\title{
Cell packing structures
}

\author{
Helmut Pottmann ${ }^{1,2}$, Caigui Jiang ${ }^{1}$, Jun Wang ${ }^{1}$, Philippe Bompas, Johannes \\ Wallner ${ }^{3}$ \\ ${ }^{1}$ King Abdullah University of Science and Technology, Thuwal 23955, Saudi Arabia \\ ${ }^{2}$ Vienna University of Technology, 1040 Vienna, Austria \\ ${ }^{3}$ Graz University of Technology, 8020 Graz, Austria
}

\begin{abstract}
This paper is an overview of architectural structures which are composed of polyhedral cells or closely related to them. We introduce the concept of a support structure of such a polyhedral cell packing. It is formed by planar quads and obtained by connecting corresponding vertices in two combinatorially equivalent meshes whose corresponding edges are coplanar and thus determine planar quads. Since corresponding triangle meshes only yield trivial structures, we focus on support structures associated with quad or hexdominant meshes. For the quadrilateral case, we provide a short survey of recent research which reveals the beautiful relations to discrete differential geometry. Those are essential for successfully initializing numerical optimization schemes for their computation. Hex-dominant structures may be designed via Voronoi tesselations, power diagrams, sphere packings and various extensions of these concepts. Apart from the obvious application as load bearing structures, we illustrate here a new application to systems for shading and indirect lighting. On a higher level, our work emphasizes the interplay between geometry, optimization, statics, and manufacturing, with the overall aim of combining form, function and fabrication into novel integrated design tools.
\end{abstract}

Keywords: architectural geometry, fabrication-aware design, spatial tiling, polyhedral packing, cell packing, polyhedral mesh, offset mesh, sphere packing, torsion free support structure, shading system

\section{Introduction}

The formation of complex materials and structures from smaller and simpler cells is an omnipresent concept in nature and certainly also in architecture. While nature is abundant in shapes of cells, architecture - at least so far - had to

Email address: helmut.pottmann (caigui.jiang, jun.wang)@kaust. edu. sa (Helmut Pottmann ${ }^{1,2}$, Caigui Jiang ${ }^{1}$, Jun Wang ${ }^{1}$, Philippe Bompas, Johannes Wallner ${ }^{3}$ ) 
be more conservative and uses simple cells which can be easily manufactured. Hence, we will deal here mostly with polyhedral cells, i.e., those which exhibit flat faces. Since part of contemporary architecture favors organic freeform shapes, we will discuss the following basic question: How can one generate freeform shells by tightly packing polyhedral cells? We will mainly focus on just one layer of cells, although we will comment on how to generate multiple layers. In the actual architectural realization, the polyhedral cells may not be completely realized or may be decorated with additional detail (see Figures 1 and 3). Moreover, we will discuss derived structures which are not really polyhedral anymore, but can easily be generated from polyhedral cell packings.
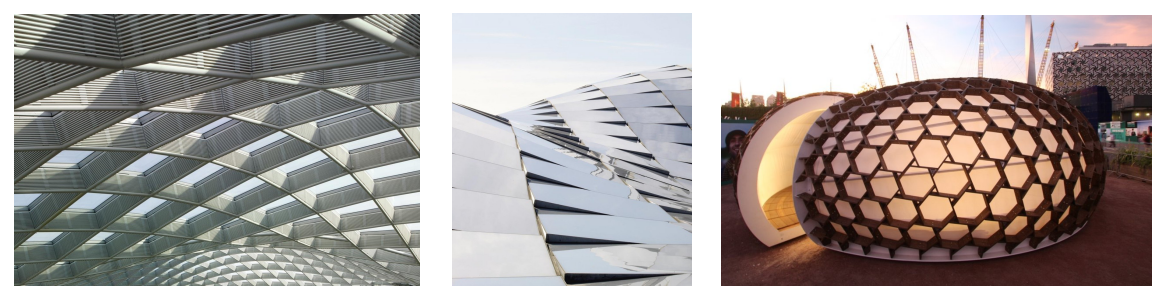

Figure 1: Real projects exhibiting cell packing structures: Left and Middle: Details from the roof of the Kogod Courtyard, Smithsonian National Portriat Gallery, Washington DC, by Foster+Partners. Right: KREOD Pavilions, by Pavilion Architecture, London (Architect: Chun Qing Li).

The present paper may be seen as a survey with new results. It covers some core developments in Architectural Geometry of the past few years, but is written from a somewhat different perspective. We therefore do not discuss prior research at the beginning of our paper, but prefer to integrate it into the relevant sections.

Our paper is organized as follows: In section 2, we formulate the problem, introduce the basic terminology and concepts and focus on cell arrangements related to Voronoi diagrams and hexagonal patterns. Section 3 presents a survey of packings with quadrilateral combinatorics and its close relations to discrete differential geometry. Aspects of statics, especially self-supporting surfaces, and the rigidity of the presented structures are discussed in Section 5. Throughout the paper, we illustrate the discussions with various applications, which are summarized and further discussed in Section 6. This leads us also to promising directions for future research.

\section{Basic types and properties of polyhedral cell structures}

A polyhedron is a solid whose boundary has planar faces only; if no confusion can arise, we will also call this polyhedral boundary surface a polyhedron. The polyhedra shall be tightly packed in order to form a thick shell (see Fig. 2). Shared faces of neighboring polyhedra will be called inner or transversal faces; the other faces are boundary faces. Analogously, a polyhedral packing has inner edges and boundary edges. 
Figure 2: Todo: Introducing the basic terminology

The support structure of a polyhedral cell packing. Packing polyhedra in this way, the planes of the inner faces, confined by the lines of the inner edges, form an important structure for our considerations. As we want the packing to have nonzero thickness everywhere, each inner face contains two inner edges, which we can connect to a quad (see Fig. 2). The resulting structure of quads will be called the support structure of the polyhedral cell packing. This name also indicates an important application: One can align prismatic beams of a load bearing structure with its faces (see e.g. Fig. 1, left). Then one speaks of a torsion-free support structure and calls the inner edges node axes.

Polyhedral plate structures. In some cases, it may be possible to close all cells of the support structure with just two flat faces, one on either side. Then we have two combinatorially equivalent polyhedral meshes $A, B$, which form the boundary of a structure we refer to as a polyhedral plate structure. It is obtained by joining corresponding vertices and edges in meshes $A$ and $B$ (which have the particular property that corresponding edges are co-planar). One may see meshes $A$ and $B$ as variable distance offsets. A practically useful special case occurs when corresponding faces of $A$ and $B$ lie in parallel planes; then also corresponding edges are parallel. Such offset meshes have been investigated in detail (see e.g. [1]). If the plates are of constant thickness (width) $w$, the planes of the support structure are bisecting planes of the adjacent face planes of the plates and the two meshes $A$ and $B$ are so-called conical meshes introduced by Liu et al. [2]. At each vertex $v_{A}$ of $A$, the bisecting planes of the incident edges of $A$ intersect in an inner edge (node axis) $n_{A}$ : Hence, $n_{A}$ is the axis of a right circular cone which touches all face planes meeting at $v_{A}$. Note that a polyhedral mesh whose vertices are all of valence three, is always conical and thus suitable for generating a polyhedral plate structure of constant thickness.

Boundary meshes $A$ and $B$ of a support structure generate an arrangement similar to the vertices and edges in a planar tiling. Thus, the basic regular types can be taken from the planar case: triangular, quadrilateral and hexagonal. In the plane, we have regular tilings all whose tiles and vertex stars are congruent. This is clearly not possible for general freeform shapes, even not on the combinatorial side. For example, instead of a regular quad tiling, the inner faces will be arranged in a quad-dominant structure, which may exhibit extraordinary vertices (valence $\neq 4$ ) and extraordinary faces (non-quads).

Triangular structures. If the support structure is arranged in a triangular way, all inner edges (node axes) pass through a common point or are parallel. This is obvious, since (i) the three face planes of a triangular cell intersect in a common point $C$ or are parallel and thus its three inner edges pass through $C$ or are parallel, and (ii) neighboring polyhedra share two inner edges. Reformulating this property, we can say that a triangle mesh does not possess a torsion-free support structure. In the present context, this is the least interesting case which will not be further pursued. 
Quadrilateral structures. A quadrilateral support structure is geometrically very interesting, and its discussion depends a bit on whether is is a plate structure or not. The quad structure in Fig. 1, left, is obviously not a plate structure, as is seen clearly in the detail of its upper side in Fig. 1, middle. We will discuss the remarkable geometry of quadrilateral structures in section 3 .
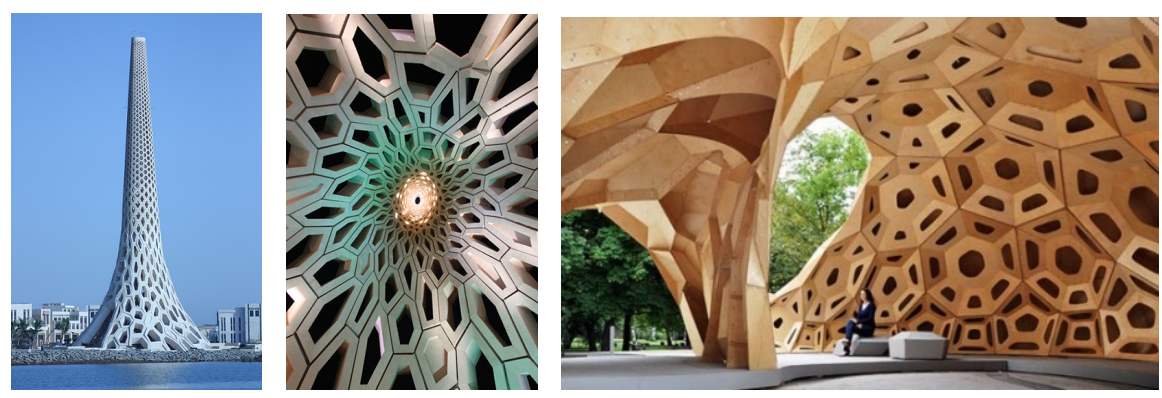

Figure 3: Hex-dominant structures. Left and Middle: The KAUST Breakwater Beacon at the entrance to the harbor of the King Abdullah University of Science and Technology in Saudi Arabia, by Urban Art Projects. Right: ICD/ITKE Research Pavilion 2011 at the University of Stuttgart.

Hexagonal structures. Such structures seem to appear frequently in nature and are closely related to Voronoi diagrams. We will discuss them below.

\subsection{Voronoi diagrams}

Given a set of seed points $s_{i}$, a Voronoi diagram associates with each of these points a cell $C\left(s_{i}\right)$ formed by all points which are closer to $s_{i}$ than to any other seed point $s_{k}$. For our application, we can place the seeds $s_{i}$ on a surface $\Phi$ and consider only that part of the Voronoi diagram which is sufficiently close to $\Phi$. This determines immediately the support structure of a polyhedral cell packing, whose nodes are generically of valence three and whose faces tend to be hexagonal, especially if the cells are roughly of the same size.

A good way to achieve nearly the same size for all cells, is to use a centroidal Voronoi tesselation (CVT) [3]. In the plane or for a full 3D diagram, this means that the seeds are the barycenters of their cells. For a diagram on a surface $\Phi$, the seeds $s_{i}$ are placed by an optimization algorithm. With $S$ as the set of seeds and $V_{i}$ as cells obtained by restricting the Voronoi diagram $V$ of $S$ to the surface $\Phi$, such a CVT minimizes

$$
E(S, V)=\sum_{i} \int_{V_{i}}\left\|p-s_{i}\right\|^{2} d p .
$$

We refer to Yan et al. [4] and for fast computation to Liu et al. [5]. Figure 4 shows examples of such structures.

In order to vary the size of the cells in a controlled way, one can use weighted Voronoi diagrams, which are also called power diagrams [6]. A seed $S_{i}$ is now a 

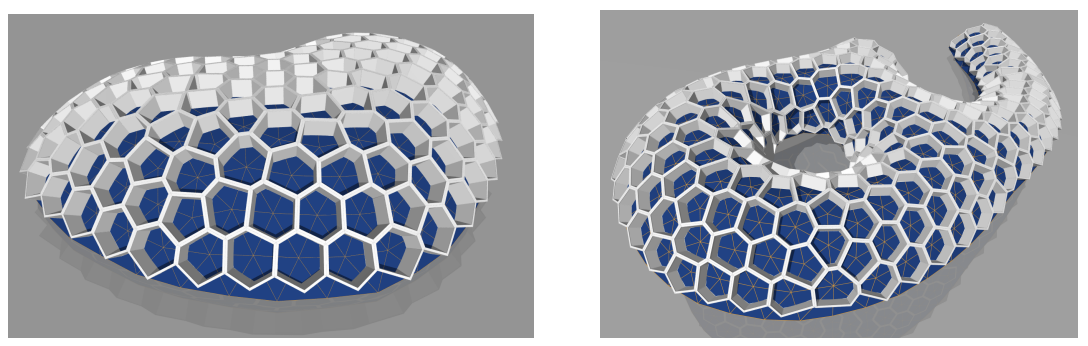

Figure 4: Hexahedral support structures of cell packings derived from centroidal Voronoi tesselations on surfaces.

sphere of a certain radius and its cell $C\left(S_{i}\right)$ is the set of all points whose power with respect to $S_{i}$ is smaller than the power with respect to any other seed sphere $S_{k}$. Recall that the power of a point $p$ with respect to a sphere with center $c$ and radius $r$ is defined as $(p-c)^{2}-r^{2}$. All points which have equal power with respect to two spheres lie in a plane, the so-called power plane. As a consequence, the power planes to three spheres (with non-collinar centers) pass through a line, and four general spheres generate 6 power planes all of which pass through a point (power center). This is used to show that the cells defined via the power of the seed spheres are polyhedral. Obviously, if the spheres are all of the same radius, power planes are bisecting planes and we get an ordinary Voronoi diagram.

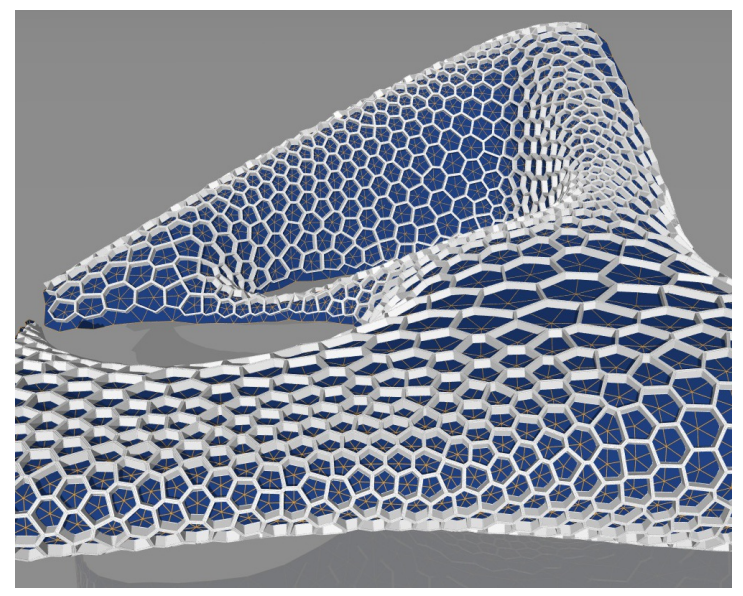

Figure 5: Cell structure based on weighted CVT (the reference surface for this illustration is a model of the Heydar Aliyev Cultural Center in Baku, by Zaha Hadid Architects).

The concept of a CVT can be extended to a weighted CVT. One prescribes a density function $\rho(p)$ on the input domain (part of 3-space) and seeks a weighted CVT where each seed sphere center lies at the barycenter of the respective 
cell and the seed sphere radius is given by the density function. Using the variational formulation, one can extend this also to surfaces by replacing the integrand in (1) by $\rho(p)\left\|p-s_{i}\right\|^{2}$ and thus generate hex-dominant patterns where the designer can steer the cell sizes (see $[4,5]$ and for an example, Fig. 5).

\subsection{Sphere packings and associated cell packing structures}

A special case of power diagrams arises when the seed spheres are tangent to the planes of their cells. This amounts to tightly packing spheres which are centered at a surface and provides us with a good way of designing support structures with well shaped, nearly 'circular' cells. Schiftner et al. [7] have discussed the basic geometry and construction of such structures.

We start with an initial triangle mesh whose combinatorics remains fixed but whose vertices $v_{i}$ are changed in the proximity of the given reference surface $\Phi$ during optimization. At each vertex $v_{i}$ we place a sphere of radius $r_{i}$; some of the radii may be fixed, but most will change during optimization. The centers of adjacent spheres are connected by an edge of the mesh. To achieve tangency of adjacent spheres and thus a sphere packing, we obviously have to minimize the following objective function,

$$
f_{p}=\sum_{\text {edges } v_{i} v_{j}}\left(\left\|v_{i}-v_{j}\right\|-r_{i}-r_{j}\right)^{2} .
$$

To make sure that vertices stay close to the reference surface $\Phi$ and boundary vertices remain close to the boundary $\partial \Phi$, we use penalty functions $f_{\text {prox }}$ and $f_{\text {prox }}^{\partial}$ as follows. Let symbols $\pi$ and $\widetilde{\pi}$ denote the closest point projections onto the surface $\Phi$ and its boundary $\partial \Phi$, respectively. Further, let $\tau_{\mathbf{x}}$ denote $\Phi^{\prime}$ s tangent plane in the point $\mathbf{x}$, and $T_{\mathbf{x}}$ denote the tangent of the boundary curve $\partial \Phi$. We let

$$
f_{\text {prox }}=\sum_{\text {vertices } v_{i}} \operatorname{dist}\left(v_{i}, \tau_{\pi\left(v_{i}\right)}\right)^{2}, f_{\text {prox }}^{\partial}=\sum_{\text {bdry vertices } v_{i}} \operatorname{dist}\left(v_{i}, T_{\widetilde{\pi}\left(v_{i}\right)}\right)^{2} .
$$

The resulting optimization problem " $F=f_{p}+\lambda_{1} f_{\text {prox }}+\lambda_{2} f_{\text {prox }}^{\partial} \rightarrow \min$ " is a nonlinear least squares problem which can be solved with a damped GaussNewton method; for details, see [7].

We consider a mesh triangle $T_{i j k}$ with vertices $v_{i} v_{j} v_{k}$ and the pairwise tangent spheres $S_{i}, S_{j}, S_{k}$. Then, the spheres' tangent planes at their contact points intersect in the rotational axis of the triangle's in-circle. This implies that the incircles of the mesh faces form a packing in the sense that the circles of adjacent faces share the same contact point at the common edge; the mesh is therefore called a circle packing $(C P)$ mesh. The support structure associated with a $\mathrm{CP}$ mesh has the in-circle axes as its node axes. A CP mesh and its sphere packing, in-circle packing and support structure are illustrated in Fig. 6.

Design flexibility. There is numerical evidence for an intuitively plausible conjecture, namely that the natural correspondence between combinatorially equivalent $\mathrm{CP}$ meshes approximates a conformal mapping of surfaces. Employing results of conformal geometry (see e.g. [8]), one then comes to the conclusion 


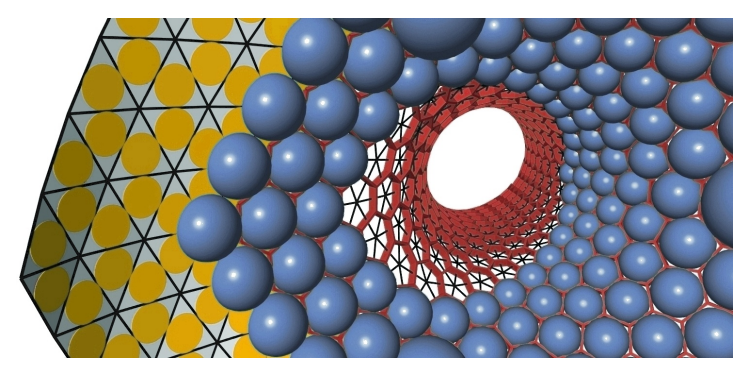

Figure 6: A CP mesh is a triangle mesh whose in-circles (orange) form a packing. It is always associated with a packing of spheres (blue), which are centered at mesh vertices. The axes of in-circles define the node axes of a hexagonal support structure whose faces lie in the tangent planes at contact points of touching spheres.
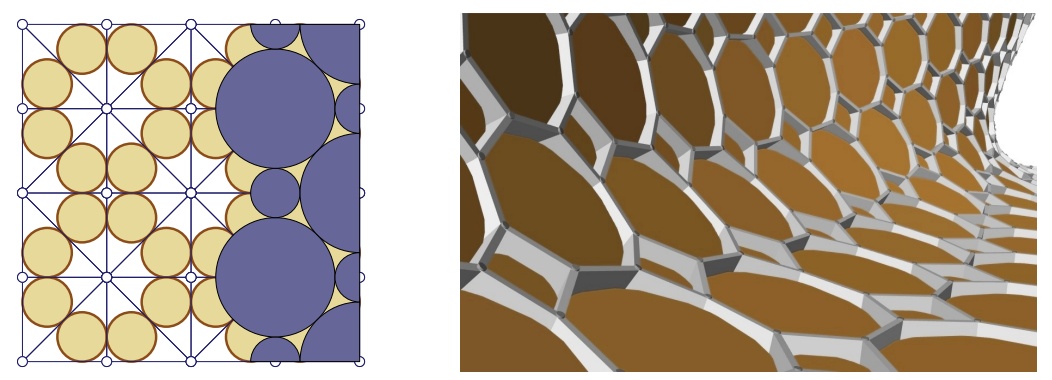

Figure 7: The connectivity of a CP mesh determines the sphere radii and the cell sizes of the resulting support structure. Left: A CP mesh with vertices of valences 4 and 8 , with associated sphere packing. Right: Derived quad-octagon support structure.

that optimization of a triangle mesh towards the $\mathrm{CP}$ property works if the reference geometry is a topological disk or topological sphere. Convergence (on closed surfaces or with fixed boundaries) is unlikely to happen in other topological cases [7]. A nice feature of CP meshes and the associated cell structures is the good control of the combinatorics. In a CVT-based cell structure, nonhexagonal cells may pop up in an undesired way, but one has the advantage of no topological restrictions (which also get weakened in CP-meshes if one adds freedom at boundaries).

\subsection{Designing anisotropic cell packings}

Much of the work discussed so far can be extended to design cell packings whose cells exhibit a given anisotropy. Geometrically, we can say that the cells on the reference surface approximate the shapes of prescribed ellipses. Hence, instead of using spheres, one uses ellipsoids. This amounts to prescribing a $3 \times 3$ tensor field $M(x)$, at least in the proximity of the reference surface. Then 
the cell centered at a point $v$ should be close to the ellipsoid with equation

$$
(x-v)^{T} \cdot M(v) \cdot(x-v)=1 .
$$

This ellipsoid can be seen as a unit sphere of a local Euclidean metric which measures squared lengths of vectors $y$ by $\|y\|^{2}:=y^{T} M(v) y$.

It has to be expected that $\mathrm{CP}$-meshes and associated sphere packings can be extended towards anisotropic cell packings as follows. We put the ellipsoid size distribution into the tensor field $M(x)$ which is seen as input. Typically one will prescribe a few tensors and obtain the field by interpolation (for tensor field design, see also Zhang et al. [9]). To get nice hexahedral cells, one would now start with a triangle mesh of regular combinatorics and optimize it so that its edges are of nearly equal length in these local metrics (which actually differ a bit at the two vertices of each edge). Hence we replace (2) by

$$
f_{p}=\sum_{\text {edges } v_{i} v_{j}}\left[\left(v_{i}-v_{j}\right)^{T} \cdot M\left(v_{i}\right) \cdot\left(v_{i}-v_{j}\right)+\left(v_{i}-v_{j}\right)^{T} \cdot M\left(v_{j}\right) \cdot\left(v_{i}-v_{j}\right)-2 c\right]^{2} .
$$

The desired squared edge length $c$ is also subject to optimization.

Todo: try out!

Shimada [10] formulates the problem of finding anisotropic cells as an ellipsoid packing problem. Li et al. [11] extended centroidal Voronoi diagrams on surfaces to the anisotropic case, but their discussion would go beyond the scope of our article.

\subsection{Hex-dominant plate structures}

In general, it is not possible to close each cell in a hex-dominant support structure with two faces, one on either side, to obtain a polyhedral plate structure. Using one plate per cell, we will see misalignment on the outside. This misalignment can be a design element (Todo: rendering with hexagonal stone cells like facades of buildings in the Middle East). Certainly, there is a variety of options to arrive at a polyhedral cell packing by using more faces per cell. This is illustrated in Fig. 8 and in Fig. 3, right (for details on this bio-inspired structure, see Knippers et al. [12]).

To design a polyhedral plate structure with hex combinatorics, one has to start from a polyhedral hex mesh. Their computation appears at first sight easy since a Phex mesh can be obtained by intersecting tangent planes at the vertices of a triangle mesh [13]. A vertex of valence 6 in the triangle mesh thus generates a hexagon of the tangent plane mesh. This approach has various shortcomings, including numerical instability, high sensitivity and lack in aesthetics. An improvement can be achieved by slightly deviating from tangent planes based on a variational formulation [14]. However, the problem is even harder: if one wants to have aesthetically pleasing faces, which cannot be convex in negatively curved areas of an underlying reference surface $\Phi$ (see Fig. 9, right), one should base the design of the hex mesh on a careful curvature analysis of $\Phi$, since the hexagons approximate the shapes of $\Phi$ 's Dupin indicatrices (see [15]). The advantage of polyhedral meshes with planar faces and vertices of only valence 3 is that they always define plate structures of constant thickness (see Fig. 9). 

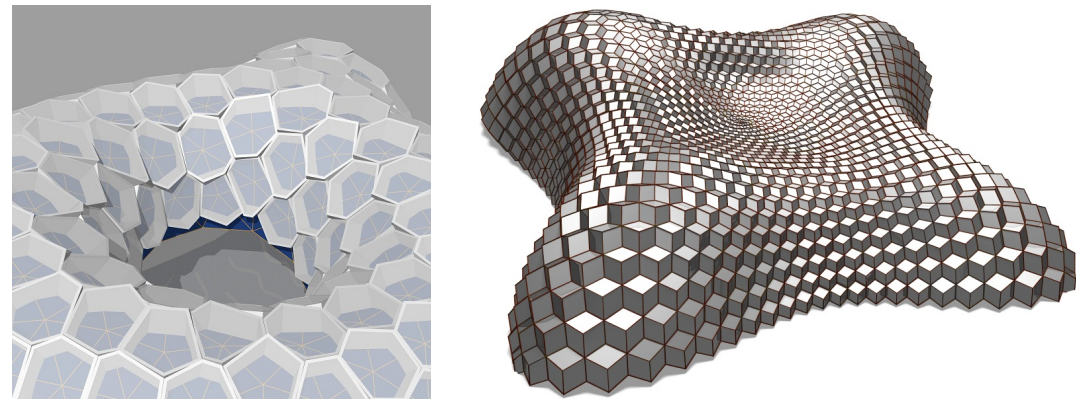

Figure 8: Left: A hexahedral support structure can in general not be extended to a hexagonal plate structure. Closing each cell just with a single plane on either side of the shell, results in misalignment which is particularly apparent in negatively curved areas of the reference surface. Right: A hexahedral support structure, derived from a CP mesh and extended towards a honeycomb-like polyhedral cell structure.
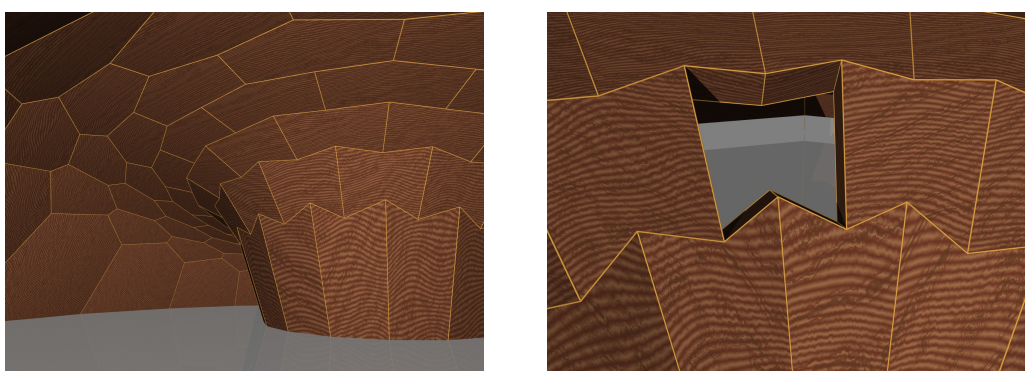

Figure 9: Hex-dominant plate structure of constant width.

\subsection{Basic patterns via parameterization algorithms}

Arbitrary patterns on surfaces can be generated by parameterization algorithms. Parameterization is of central importance for texture mapping in Computer Graphics and thus it has been studied extensively . Its discussion within the present context would lead too far. However, as we have dealt here a lot with hexagonal patterns, we refer to the work of Nieser et al. [16] on hexagonal global parameterization; this paper also contains a long list of references on parameterization.

\subsection{Computing a support structure to a mesh}

Given a mesh $A$ with vertices $a_{i}$, can we find a mesh $B$ with vertices $b_{i}$ so that they define a support structure? This associates a constraint with each edge $a_{i} a_{j}$, namely planarity of the quad $a_{i} a_{j} b_{j} b_{i}$. We have one degree of freedom for the plane of this quad. At a vertex of valence $v$, all these planes have to be co-axial, which amounts to $v-2$ scalar constraint equations. 
A support structure to a mesh all whose vertices have valence 3 , gives rise to one degree of freedom per face and one scalar condition per vertex. Leaving aside a possible boundary, the numbers $v, e$ of vertices and edges respectively, are related by $2 e=3 v$, and thus we have 1.5 times more degrees of freedom than constraints and an optimization algorithm will work. We can even be more specific and try to get node axes as orthogonal as possible to the reference surface (see Figures 10 and 18), or planes of the structure arranged so that they optimally block sun light for selected sun positions (Fig. 19). The formulation and implementation of the optimization algorithm follows the final optimization step suggested by Wang et al. [17] for the harder case of quad structures.
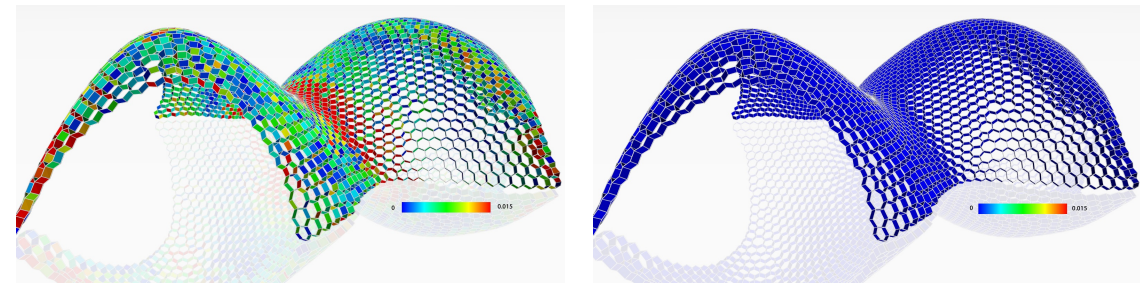

Figure 10: Computing a support structure for a hex mesh so that node axes are as orthogonal as possible to the reference surface. Left: Initialization of the optimization with node axes as surface normals; right: after optimization towards planarity of faces. The deviation from planarity is color coded by the diagonal distance in the quads, based on a scale which sets the average edge length in the hex mesh to 1.

The above count of degrees of freedom also shows that chances to compute a support structure to a quad mesh (without allowing to adapt the mesh) are much smaller, since we have 2 constraints for a vertex of valence 4 , leading roughly to balance between the number of unknowns and degrees of freedom. This is not sufficient if the support structure has to satisfy additional constraints like orthogonality to a reference surface or blocking of sun. Fortunately, as discussed in the next section, the quadrilateral case is deeply rooted in differential geometry and this helps us to succeed.

\section{Quad-dominant structures}

\subsection{Structures from planar quad meshes}

Todo: very short, since addressed well in other papers. Only the basic insight is important here.

PQ; integrable systems and DDG [18] appearance of special cases in architecture: Glymph [19] conical [2], multi-layer [1] support structures arise through parallel meshes.

Further references to PQ meshes and more general polyhedral meshes: Zadravec et al. [20], Liu et al. [21], exploration [22], alternative computations [23, 24], Vaxman [25] linear spaces in the space of polyhedral meshes [26] 


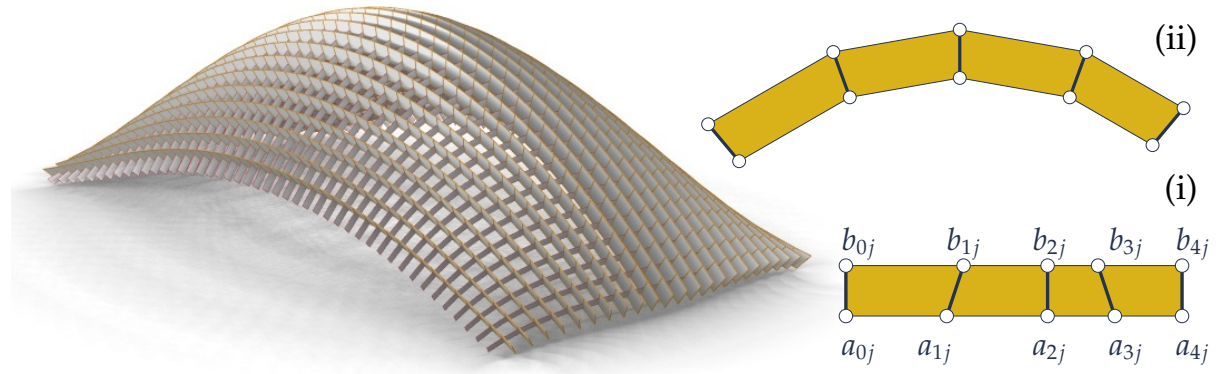

Figure 11: A quadrilateral support structure defines prismatic beams of constant height along a mesh polyline $\left\{a_{i, j}\right\}_{j=\text { const }}$ in exactly two cases: (i) Unfolding the discrete developable through the mesh polyline either yields a straight strip, or (ii) a strip where the lines connecting $a_{i, j}, b_{i, j}$ are bisecting the angle between adjacent edges. The example at left has constant beam height along one family of beams (the larger one), it belongs to case (ii).

sphere packing with quad combinatorics: discrete isothermic surfaces: Bobenko et al. [27], approximately isothermic [28].

Design flexibility. shortly discuss the degrees of freedom for the important cases.

3.2. Support structures for non-polyhedral quad meshes: Discrete torsal parameterizations of line congruences

Todo: Very short description of the connection to line congruences and reference to DDG Doliwa [29] and our SGP paper [17].

Relation to support structures associated with $P Q$ meshes. Into a quad support structure $S$, we can (at least locally) fit a PQ mesh $M$ whose vertices lie on the node axes of $S$. To illustrate this claim, let us take a simply connected quad support structure $S$ with grid combinatorics. Then, we can choose two transversal polylines of the PQ mesh aligned with $S$ and the rest follows by planarity of faces, since each vertex of the PQ mesh is constrained to a given node axis of $S$. However, this construction does not have enough degrees of freedom to maintain proximity to a reference surface. Likewise, as discussed above, if we first compute the PQ mesh, we usually do not have enough degrees of freedom to attach a support structure which satisfies additional requirements. Hence, if we are just interested in a quad support structure and not in a PQ mesh attached to it, it is not a good ideas to base its construction on a PQ mesh and the related concept of parallel meshes.

Beams of constant height. The question if edges of meshes $A, B$ with planar faces can be at constant distance was studied by [1]. It turns out that $A$ must have special properties. For a general mesh $A$ lying on a discrete torsal parametrization we can always achieve parallel edges along one family of mesh polygons, but not for both. In this one family one can achieve constant height of the beams in exactly two cases which are illustrated by Figure 11. 


\section{Design flexibility. Todo}

Multiple layers. Todo Straightforward for Voronoi-based cell packings. Extension of quad structures to 3D, i.e. hexahedral cells with planar faces, see [18]. Computational approach in our paper on circular arc structures, but not yet fully developed.
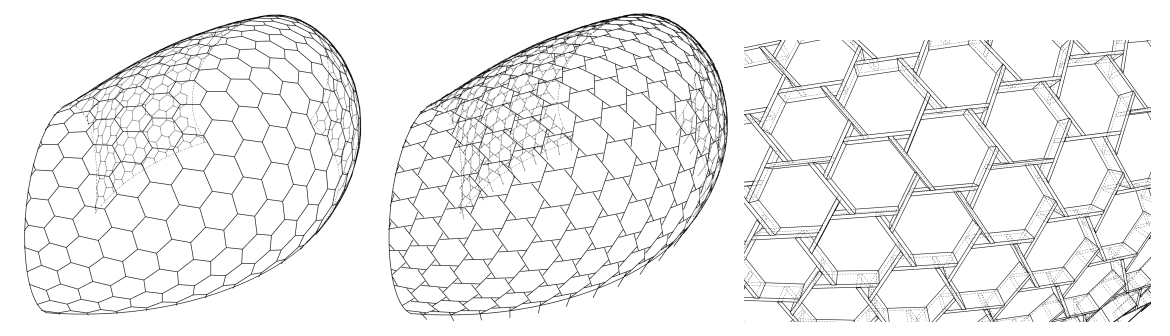

Figure 12: A CP mesh generates a hex-mesh (left), whose edges are rotated so as to form nearly equally sized triangular nodes (middle) along which the beams of a reciprocal structure for the Kreod Pavilion are placed (right); images courtesy Evolute, Vienna.

\section{Derived structures}

Resolvig nodes and reciprocal structures. There are many ways to derive further structures from those we have discussed so far. Given a support structure, one may resolve its node axes into cells either by an appropriate rotation or translation of the face planes of cells. An example is provided by Fig. 12, where the nodes of valence 3 in the original structure become triangular cells. They are stable and easier to be manufactured from wooden elements than the original nodes. The resulting design shares some basic geometry with reciprocal frame structures. For a classical architectural discussion of reciprocal structures, see [30]; a very recent computational design approach is due to Song et al. [31].

There is obviously a close connection between planar tilings and patterns arising in reciprocal frame structures (for another example based on a quad mesh, see Fig. 13). It is not necessary to start the computation from a support structure with nodes of higher valence and resolve them; we can generate reciprocal structures directly, e.g. by parameterization algorithms (see Song et al. [31]). The challenge lies in providing the designer with handles to steer shape, orientation and size of the cells. This is part of our ongoing research and is connected to the computational design of shading structures.

Transfer of 3D textures. Basic support structures suggest a general way of generating designs made from repeating small elements. This is illustrated by Figure 14 with help of a quadrilateral support structure. It consists of many combinatorial cubes, and we can use 3D texture mapping to transfer any master element (contained in the unit cube) to each of them. 

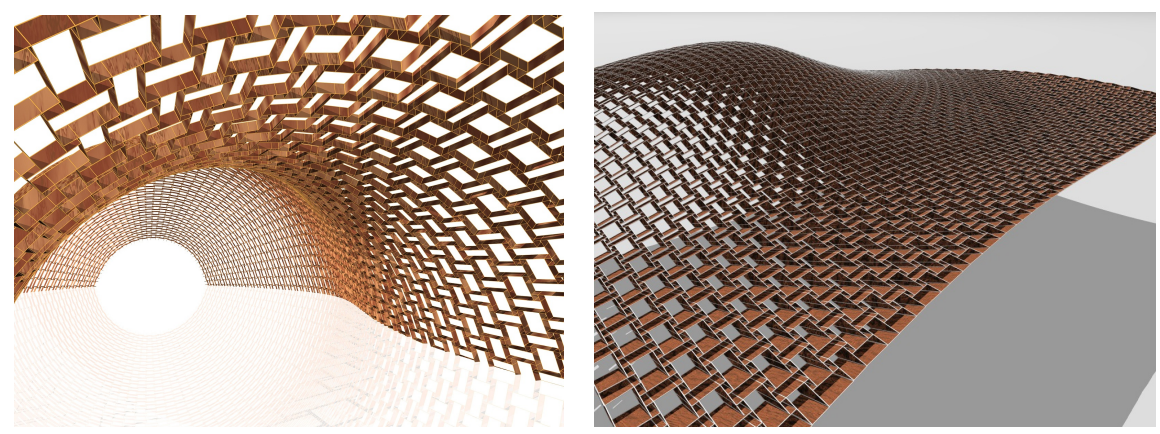

Figure 13: Reciprocal frame structures with quadrilateral cells. The structure on the right has been optimized for blocking light at selected positions of the sun.
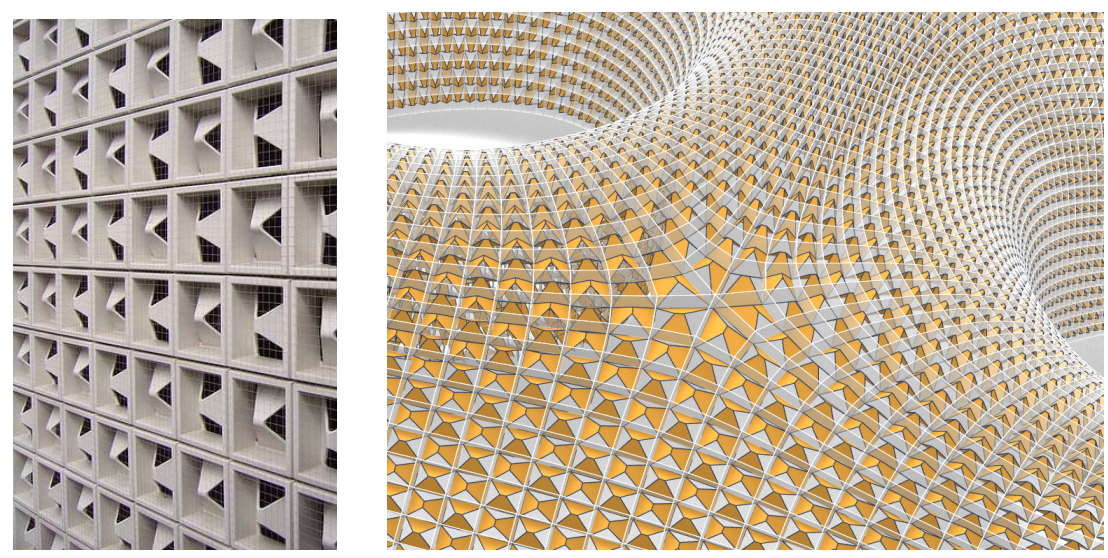

Figure 14: A design by Egon Eiermann, at left, motivates a shading system in form of a 3D texture. A quadrilateral support structure (transparent) allows us to transfer this design to a curved building skin.

Cells bounded by developable surfaces. A sequence of planar quadrilaterals is a discrete developable surface. This interpretation motivates us to apply a refinement procedure according to [2] to a support structure in order to convert it into a system of smooth developable surface strips: we iteratively apply splitting, smoothing, and optimization towards planar faces (see Fig. 15). Depending on how exactly the splitting is done, we end up with different patterns of developables - Fig. 15, top row, and Fig. 16, left, shows one which follows the mesh polylines, while Fig. 15, bottom row, Fig. Fig. 16, right, and Figure 17 exhibit one developable per cell. Applications for the former are structures built from plywood or sheet metal, whose manufacturing depends on the developability property. The latter is useful for applying known methods of lighting to curved building skins (see Figure 17; for previous work combining shading with subdivision, but such that the shading system follows the edges of 

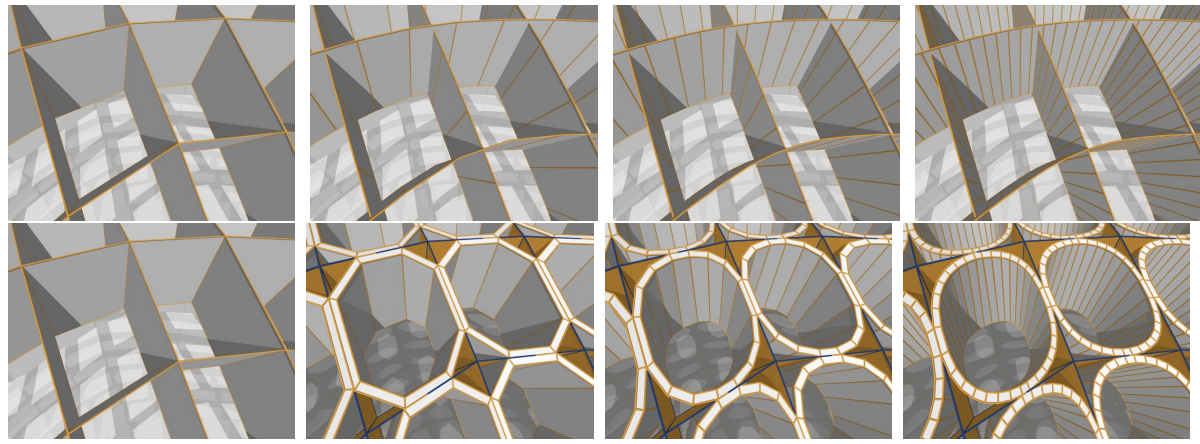

Figure 15: Subdivision of a quadrilateral support structure in direction of mesh polylines (top row) and per cell (bottom row). From left to right: Original, and result after 1,2 and 3 rounds of subdivision.
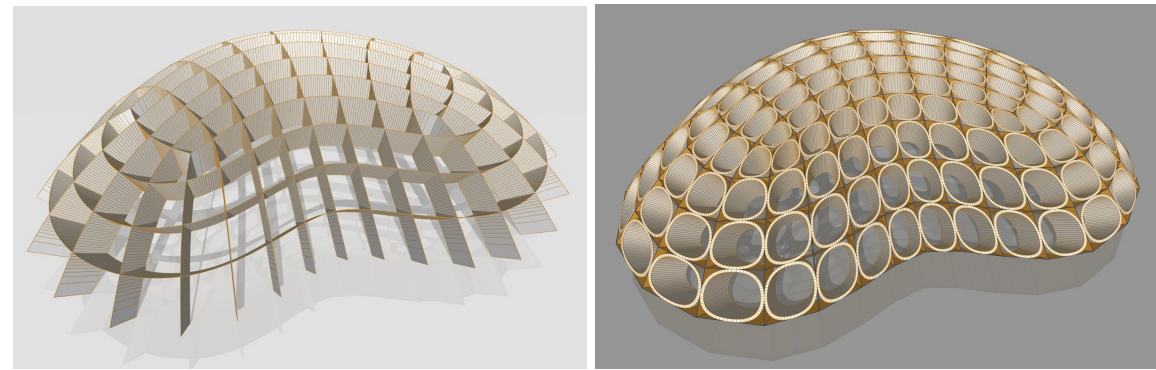

Figure 16: Packings of cells which are bounded by developable surfaces and generated from a quad support structure via subdivision along mesh polylines (left) and faces (right).

a mesh created by subdivision, see http://aedasresearch.com/features/view/all/ method/envelope-design).

\section{Rigidity and statics}

Todo: Short discussion of thrust networks and design of self-supporting masonry structures $[32,33,34,35,36,37]$. Hexahedral cell packing (bricks) in particular in the paper by Panozzo et al [36].

Torsion-free support structures attached to PQ meshes and in static equilibrium [37], formfinding with polyhedral meshes and associated support structures [38]

Infinitesimal flexibility test through velocity fields. Address stability of nodes in reciprocal structures.

Todo: Example of rigidity analysis for a reciprocal structure with quad combinatorics. We can show that the system gets more rigid as the four axes 

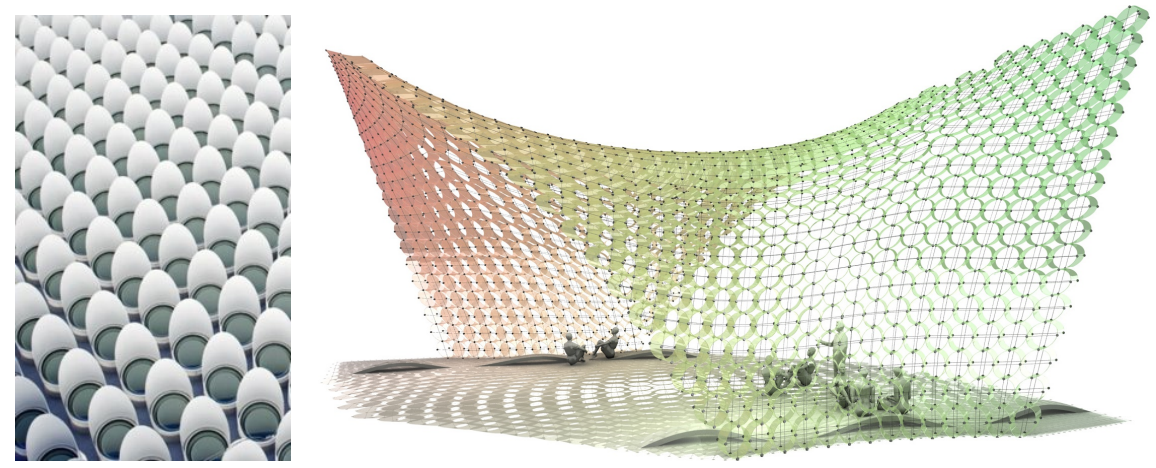

Figure 17: Indirect lighting by oblique cylinders (at left, High Museum of Art, Atlanta) motivates a shading system consisting of developables, created by a subdivision process.

in a node do not lie in a bundle. Similar to our paper for the Design Modeling Symposium.
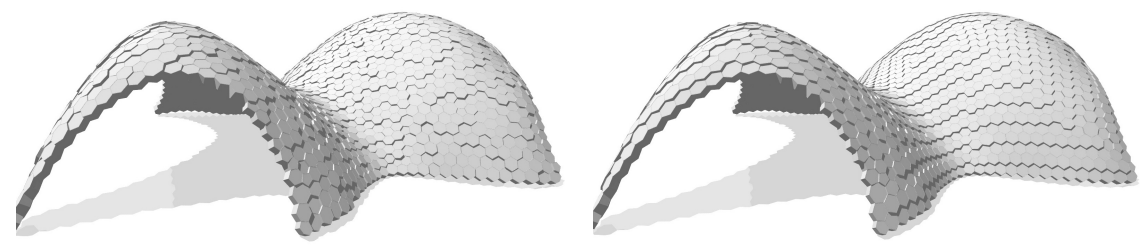

Figure 18: Hexagonal structures for a self-supporting surface. The underlying support structure is that of Fig. 10, right. The mis-alignment of plates can be used for the generation of a pattern which becomes particularly visible through light and shade effects. The two examples differ in the height computation for the hexagonal cells, giving rise to different patterns.

\section{Applications and Discussion}

There are numerous applications of cell packing structures, simply because it is in most cases not feasible to build a large structure in a monolithic way. One has to decompose it into smaller units, which naturally leads to some kind of cell packing. Obviously, the geometric concept of support structures as discussed in our paper, is suitable for load bearing structures, and has been used for that in many real projects, espcially steel/glass constructions. Cell packing structures, in particular those which are statically optimized (such as the ones in Fig. 18), may be built from opaque material like stone, leading to contemporary freeform designs from natural and maybe often locally available material. 
A wide and not yet systematically studied application area is the use of cell packing structures for shading and indirect lighting (see Wang et al. [17] and Figures 20 and 19).

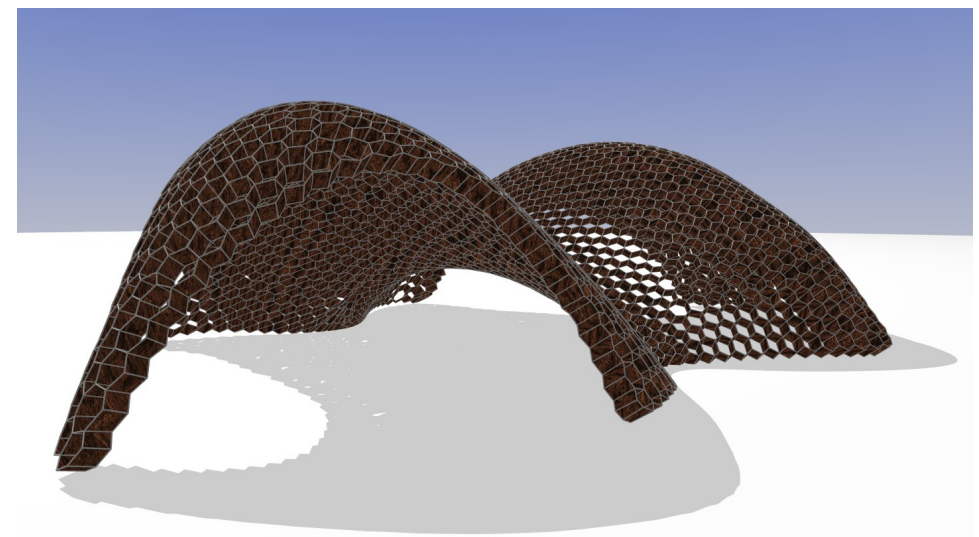

Figure 19: In this example, the hex mesh of Fig. 10 has been extended to a support structure which blocks light for a given light direction and thus is suitable as a shading structure.

\section{Conclusion and future work}

We have provided an overview of architectural structures which are composed of polyhedral cells or closely related to them. As this is a wide topic, our paper is certainly biased towards work in Architectural Geometry, especially by researchers from Geometry Processing. We added new aspects at various places, having in mind the overall goal of combining form, function and fabrication into novel integrated design tools.

We have very briefly touched our own ongoing work on the theme of this paper, which develops around structurally sound shading systems based on reciprocal meshes in a design environment which leaves enough artistic freedom and helps to keep the structure as light and cost-effective as possible.

It is hoped that our discussion illustrated the necessity to carefully elaborate on the relationships between geometry, numerical analysis and aspects of statics, functionality and manufacturing. In this area, we have so far only scratched the surface, and there are plenty of possibilities for future research.

\section{Acknowledgments}

This research was supported in part by the DFG-Collaborative Research Center, TRR 109, Discretization in Geometry and Dynamics, through grant I 706N26 of the Austrian Science Fund (FWF). We would like to thank Dongming Yan for his help with the CVT examples and Chengcheng Tang for providing the hex mesh to Fig. 9. 


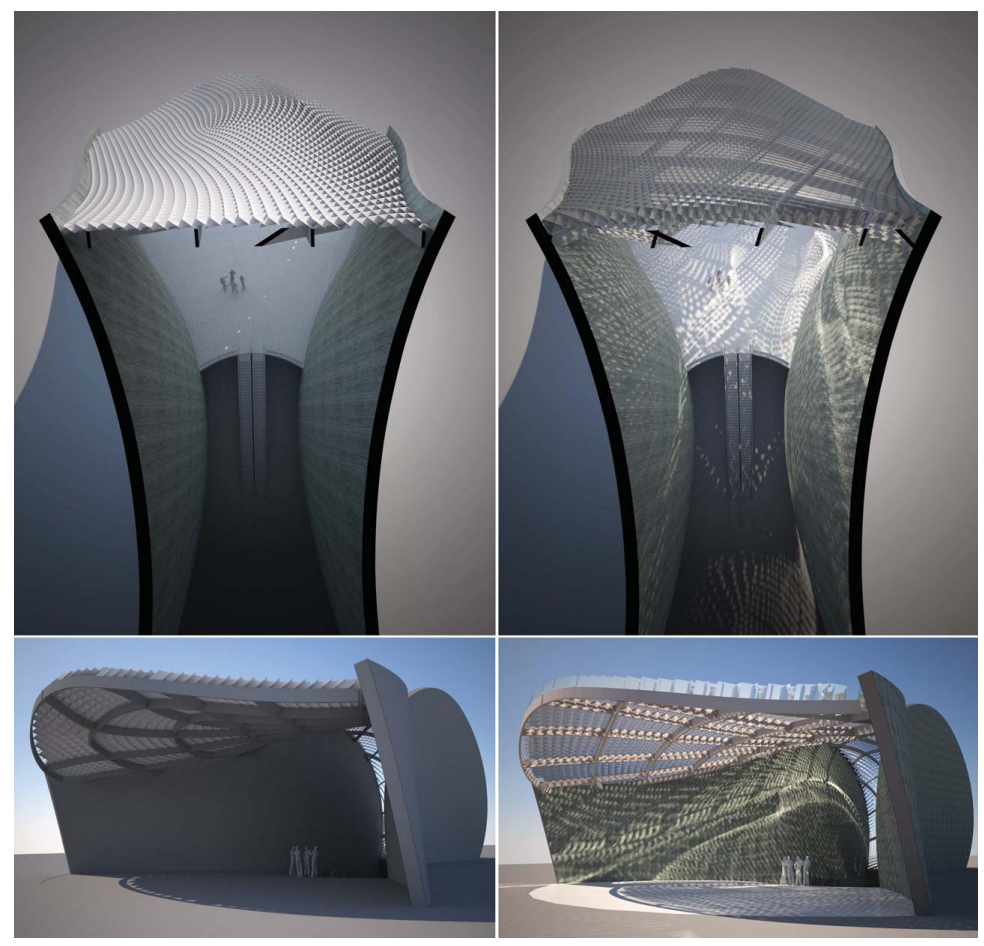

Figure 20: Two aligned quadrilateral support structures, one for load bearing and, on top of it, one for shading or indirect lighting. Left: The top structure is optimized for shading during the hottest part of June 21st (location: London). Right: The top structure blocks direct sunlight, but reflects light into the entrance hall of this subway station. For details on the computation of such structures we refer to Wang et al. [17]

[1] H. Pottmann, Y. Liu, J. Wallner, A. Bobenko, W. Wang, Geometry of multilayer freeform structures for architecture, ACM Trans. Graphics 26 (3) (2007) \#65,1-11. doi : 10 .1145/1275808. 1276458.

[2] Y. Liu, H. Pottmann, J. Wallner, Y.-L. Yang, W. Wang, Geometric modeling with conical meshes and developable surfaces, ACM Trans. Graphics 25 (3) (2006) 681-689. doi : 10.1145/1141911.1141941.

[3] Q. Du, V. Faber, M. Gunzburger, Centroidal Voronoi tessellations: applications and algorithms, SIAM Review 41 (1999) 637-676.

[4] D.-M. Yan, B. Lévy, Y. Liu, F. Sun, W. Wang, Isotropic remeshing with fast and exact computation of restricted Voronoi diagram 28 (5) (2009) $1445-1454$. 
[5] Y. Liu, W. Wang, B. Lévy, F. Sun, D.-M. Yan, L. Lu, C. Yang, On centroidal Voronoi tessellation - energy smoothness and fast computation, ACM Trans. on Graphics 28 (4) (2009) Article No. 101.

[6] F. Aurenhammer, Power diagrams: Properties, algorithms and applications, SIAM J. Comput. 16 (1) (1987) 78-96.

[7] A. Schiftner, M. Höbinger, J. Wallner, H. Pottmann, Packing circles and spheres on surfaces, ACM Trans Graphics 28 (5) (2009) \#139,1-8.

[8] X. D. Gu, S.-T. Yau, Computational Conformal Geometry, International Press, 2008.

[9] E. Zhang, J. Hays, G. Turk, Interactive tensor field design and visualization on surfaces, IEEE Trans. Vis. Comp. Graphics 13 (1) (2007) 94-107.

[10] K. Shimada, A. Yamada, T. Itoh, Anisotropic triangulation of parametric surfaces via close packing of ellipsoids, Intl. J. Comp. Geometry Appl. 10 (2000) 417-440.

[11] H. Li, L.-Y. Wie, P. Sanders, C.-W. Fu, Anisotropic blue noise sampling, ACM Trans. Graphics 29 (2010) \#167, 1-12.

[12] J. Knippers, M. Gabler, R. L. Magna, F. Waimer, A. Menges, S. Reichert, T. Schwinn, From nature to fabrication: Biomimetic design principles for the production of complex spatial structures, in: L. Hesselgren, et al. (Eds.), Advances in Architectural Geometry 2012, Springer, 2012, pp. 107-122.

[13] C. Troche, Planar hexagonal meshes by tangent plane intersection, in: Advances in Architectural Geometry, 2008, pp. 57-60.

[14] H. Zimmer, M. Campen, R. Herkrath, L. Kobbelt, Variational tangent plane intersection for planar polygonal meshing, in: L. Hesselgren, et al. (Eds.), Advances in Architectural Geometry 2012, Springer, 2012, pp. 319-332.

[15] W. Wang, Y. Liu, D.-M. Yan, B. Chan, R. Ling, F. Sun, Hexagonal meshes with planar faces, Tech. Rep. TR-2008-13 (CS series), University of Hong Kong (2008).

[16] M. Nieser, J. Palacios, K. Polthier, E. Zhang, Hexagonal global parameterization of arbitrary surfaces, IEEE Trans. Vis. Comp. Graphics 18 (6) (2012) 865-878.

[17] J. Wang, C. Jiang, P. Bompas, J. Wallner, H. Pottmann, Discrete line congruences for shading and lighting, Computer Graphics Forum 32, proc. Symp. Geometry Processing.

[18] A. Bobenko, Yu. Suris, Discrete Differential Geometry: Integrable Structure, American Math. Soc., 2009. 
[19] J. Glymph, D. Shelden, C. Ceccato, J. Mussel, H. Schober, A parametric strategy for freeform glass structures using quadrilateral planar facets, Automation in Contruction 13 (2004) 187-202.

[20] M. Zadravec, A. Schiftner, J. Wallner, Designing quad-dominant meshes with planar faces, Computer Graphics Forum 29 (5) (2010) 1671-1679.

[21] Y. Liu, W. Xu, J. Wang, L. Zhu, B. Guo, F. Chen, G. Wang, General planar quadrilateral mesh design using conjugate direction field, ACM Trans. Graphics 30 (2011) \#140, 1-10. doi : 10. 1145/2024156. 2024174.

[22] Y. Yang, Y. Yang, H. Pottmann, N. Mitra, Shape space exploration of constrained meshes, ACM Trans. Graphics 30 (2011) \#124,1-11.

[23] S. Bouaziz, Y. Schwartzburg, T. Weise, M. Pauly, Shaping discrete geometry with projections, Computer Graphics Forum 31, proc. Symp. Geometry Processing.

[24] R. Poranne, E. Ovreiu, C. Gotsman, Interactive planarization and optimization of 3d meshes, Computer Graphics Forum 32 (1) (2013) 152-163.

[25] A. Vaxman, Modeling polyhedral meshes with affine maps, Computer Graphics Forum 31, proc. Symp. Geometry Processing.

[26] R. Poranne, R. Chen, C. Gotsman, On linear spaces of polyhedral meshes, ArXiv:1303.4110 (2013).

[27] A. Bobenko, T. Hoffmann, B. Springborn, Minimal surfaces from circle patterns: Geometry from combinatorics, Ann. of Math. 164 (2006) 231264.

[28] S. Sechelmann, T. Rörig, A. Bobenko, Quasiisothermic mesh layout, in: L. Hesselgren, et al. (Eds.), Advances in Architectural Geometry 2012, Springer, 2012, pp. 243-258.

[29] A. Doliwa, P. Santini, M. Mañas, Transformations of quadrilateral lattices, J. Math. Phys. 41 (2000) 944-990. doi : 10.1063/1.533175.

[30] O. Popovic-Larsen, Reciprocal Frame Architecture, Elsevier, 2008.

[31] P. Song, C.-W. Fu, P. Goswami, J. Zheng, N. Mitra, D. Cohen-Or, Reciprocal frame structures made easy, ACM Transactions on Graphics (proceedings of ACM SIGGRAPH) 32 (4) (2013) to appear.

[32] P. Block, J. Ochsendorf, Thrust network analysis: A new methodology for three-dimensional equilibrium, J. Int. Assoc. Shell and Spatial Structures 48 (3) (2007) 167-173.

[33] P. Block, Thrust network analysis: Exploring three-dimensional equilibrium, Ph.D. thesis, Massachusetts Institute of Technology (2009). 
[34] F. de Goes, P. Alliez, H. Owhadi, M. Desbrun, On the equilibrium of simplicial masonry structures, ACM Transactions on Graphics (proceedings of ACM SIGGRAPH) 32 (4) (2013) to appear.

[35] Y. Liu, H. Pan, J. Snyder, W. Wang, B. Guo, Computing self-supporting surfaces by regular triangulation, ACM Transactions on Graphics (proceedings of ACM SIGGRAPH) 32 (4) (2013) to appear.

[36] D. Panozzo, P. Block, O. Sorkine-Hornung, Designing unreinforced masonry models, ACM Transactions on Graphics (proceedings of ACM SIGGRAPH) 32 (4) (2013) to appear.

[37] E. Vouga, M. Höbinger, J. Wallner, H. Pottmann, Design of self-supporting surfaces, ACM Trans. Graphics 31 (2012) \#87,1-11, proc. SIGGRAPH.

[38] C. Tang, X. Sun, R. Ait-Haddou, A. Gomes, H. Pottmann, J. Wallner, Formfinding with polyhedral meshes, submitted for publication. 